\title{
The One-Dimensional, Non-Relativistic, Quanitum Morse Oscillator at the Classical Limit
}

\author{
M.A. Grado-Caffaro and M. Grado-Caffaro \\ Permanent address: M.A. Grado-Caffaro and M. Grado-Caffaro- Scientific Consultants, C/Julio Palacios 11, 9-B, \\ 28029-Madrid (Spain); www.sapienzastudies.com; \\ Email: ma.grado-caffaro@sapienzastudies.com
}

\begin{abstract}
The one-dimensional, non-relativistic, quantum Morse oscillator is studied at the classical limit. In fact, near the classical limit, the energy eigenvalues relative to the eigenstates of the nonrelativistic, time-independent, Schrödinger equation with Morse potential are negative and approximately proportional to the square of the corresponding vibrational quantum number. Within this framework, the mass of the oscillator in question is found to be negative. This can take place in certain particle phenomena and, in fact, occurs, for instance, in semiconductor superlattices. These cases are outlined very briefly in the present paper.
\end{abstract}

Keywords: Morse oscillator; classical limit; negative mass; non-relativistic quantum particles.

\section{Introduction}

The significant role of the Morse potential in Nuclear Physics and Molecular Physics is well-known. Really, the above potential has a great relevance within Particle Physics in its broad sense. Indeed, studying the behaviour of both relativistic and non-relativistic quantum particles under the Morse potential presents a wide variety of issues of much interest. It is well-known that the above (nonrelativistic) quantum anharmonic oscillators have a finite number of bound states and infinite number of unbound states. As a matter of fact, considering the one-dimensional case, the energy eigenvalues relative to the unbound states are negative and roughly proportional to the square of the involved vibrational quantum number. This corresponds to the anharmonic oscillator in question close to the classical limit so energy tends to minus infinity as the quantum number tends to infinity. But, unfortunately, in a certain part of the current literature, the existence of negative energy eigenvalues by, say, extrapolation to them of the general formula for the energy levels, is not understood.

In the following, we will show that the mass of the aforementioned Morse oscillator must be negative near the classical limit. The one-dimensional case will be regarded. In addition, as notorious and interesting examples, we will discuss the role of electrons of negative mass in semiconductor superlattices [1-3] and, on the other hand, we will comment on cosmological as well as on Bose condensates and various elementary-particle questions dealing with the possibility of negative rest-mass [4,5]. In particular, with respect to semiconducting superlattices, we note that the fact that there are electrons with negative rest-mass mass in these structures comes from the existence of allowed and forbidden minibands formed from allowed and forbidden energy bands [1-3]. In this context, there are negative differential and absolute electrical conductivities [1-3]. Really, the above mentioned facts have great importance and will be discussed very briefly.

\section{Theoretical Formulation}

The energy eigenvalues of a (non-relativistic) quantum-mechanical particle under a one-dimensional Morse potential read:

$$
E_{n}=\hbar \omega\left(n+\frac{1}{2}-\varepsilon n-\varepsilon n^{2}-\frac{\varepsilon}{4}\right)
$$

where $n$ is the corresponding vibrational quantum number $(n=0,1,2, \ldots)$ and $\varepsilon$ is an anharmonic coefficient. From formula (1) it follows that $E_{n} \rightarrow-\infty$ as $n \rightarrow \infty$ (classical limit) so that $E_{n} \approx-\hbar \omega \varepsilon n^{2}$ for sufficiently large $n$. Therefore, near the classical limit, the last expression for the quantized 
vibrational energy equals the kinetic energy of the particle plus the Morse potential energy. This last energy is non-negative and is given by:

$$
V(x)=D\left\{1-\exp \left[-\alpha\left(x-x_{0}\right)\right]\right\}^{2}
$$

where $D$ denotes potential depth, $x_{0}$ is the equilibrium coordinate of position, and $\alpha$ is a parameter that "controls" the potential width. We assume $x>0, x \geq x_{0}$.

By the energy-conservation law above and taking into account relation (2), since now $E_{n}$ is negative, then the kinetic energy must be negative with absolute value larger than $V(x)$ so it is clear that the (quantized) kinetic energy of the particle must be $-m v_{n}^{2} / 2(m>0), v_{n}$ being the magnitude of the (quantized) particle velocity, so the rest-mass of the particle must be negative and equal to $-m$. Under these conditions, for $x \approx x_{0}$ (so, by $(2)$, one has $V \approx 0$ ), considering that $\varepsilon=\hbar \omega /(4 D)$, and regarding the expression of the energy for $n$ near the classical limit, then it follows:

$$
v_{n} \approx \frac{\hbar \omega n}{\sqrt{2 D m}}
$$

We wish to remark that formula (3) refers to that the Morse potential (see eq.(2)) becomes an ideal potential near the equilibrium position of the particle. This situation is similar to that of a nonrelativistic quantum particle in a one-dimensional, infinite, ideal potential well. In effect, for this well, we have:

$$
E_{n}=-\frac{\pi^{2} \hbar^{2} n^{2}}{2 m a^{2}}
$$

where $a$ is the length of the well and now $n=1,2, \ldots$

Equating approximately (4) to $-m v_{n}^{2} / 2$ (this is only valid for sufficiently large $n$ ), then one gets:

$$
v_{n} \approx \frac{\pi \hbar n}{m a}
$$

Finally, equating (3) to (5), an equivalent angular frequency reads:

$$
\omega \approx \frac{\pi}{a} \sqrt{\frac{2 D}{m}}
$$

For non-equilibrium enough in the Morse potential, that is, for, $x>>x_{0}$, expression (2) reduces to $V \approx D$ so that, by the energy-conservation law, we have:

$$
-\frac{\hbar^{2} \omega^{2} n^{2}}{4 D} \approx-\frac{1}{2} m v_{n}^{2}+D
$$

From formula (7) it follows:

$$
v_{n} \approx \sqrt{\frac{\hbar^{2} \omega^{2} n^{2}-4 D^{2}}{2 D m}}
$$

On the other hand, inserting (6) into (3), one finds (5) as expected, while inserting (6) into (8), we get:

$$
v_{n} \approx \frac{\sqrt{\pi^{2} \hbar^{2} n^{2}-2 D m a^{2}}}{m a}
$$

At any rate, for $n$ values such that $\pi^{2} \hbar^{2} n^{2}>2 D m a^{2}$, formula (9) reduces to (5). In practice, the above inequality is satisfied for every quantum particle and, say, typical potential-well lengths if $n$ is sufficiently large. Therefore, we conclude that the particle velocity near its equilibrium position equals approximately the velocity far from the equilibrium position (consider formula (5)).

The importance of our preceding formulation is significant to investigate, for instance, certain problems concerning Cosmology and Particle Physics [4]. In this context, negative mass can exist, for example, in non-asymptotically flat space-time [4]. On the other hand, negative mass has been found in a Bose condensate fluid [5]. Finally, we can find negative effective electron mass in semiconductor superlattices [1-3]. In these structures, allowed and forbidden bands are divided into a set of relatively narrow allowed and forbidden minibands with non-parabolic dispersion law. In a part of a given 
miniband, the electron effective mass can change its sign so that it becomes negative [1-3]. Consequently, the superlattices may present nonlinear properties even under relatively small electric fields [1-3].

\section{Conclusions}

The preceding analysis shows that particles with negative mass can exist under nonlinear conditions, for instance, in certain semiconductor structures [1-3]. In fact, we have proven this (within the framework of the Morse potential) by calculating the quantized particle velocity, arriving at formulae (3), (5), (6) and (9) as main results. It is clear that our formulation arises from considering the quantized particle energy (see relationship (1)) when the corresponding quantum number is sufficiently large so that the energy becomes negative. This extrapolation to (non-relativistic) quantum states of negative energy leads to the existence of negative mass. On the other hand, we should remark that a relativistic quantum formulation of the same, say, type as our formulation could be useful to explain phenomena as described in refs.[4,5]. Finally, one would mention refs.[6,7] as valuable work related indirectly to the present paper.

\section{References}

1. Yu.A. Romanov, Yu.Yu. Romanova, Phys. Solid State 43 (2001) 539.

2. Yu.A. Romanov, Phys. Solid State 45 (2003) 559.

3. Yu.Yu. Romanova, Semiconductors 49 (2015) 1557-1563.

4. S. Mbarek, M.B. Paranjape, Phys. Rev. D 90 (2014) 101502 (R).

5. M.A. Khamehchi, K. Hossain, M.E. Mossman, Y. Zhang, Th. Busch, M.McNeil Forbes, P. Engels, Phys. Rev. Lett. 118 (2017) 155301.

6. A. Soldatov, N. Bogolyubov (Jr.), S. Kruchinin, Quant. Matter 4 (2015) 352-357.

7. S. Kruchinin, Int. J. Mod. Phys. B 30 (2016) 1042008. 